\title{
Outcome of endoscopic carpal tunnel release versus open carpal tunnel release in diabetic patients: a randomized controlled prospective double blinded study
}

\author{
Mohamed Said Abdelmaksoud Mohamed Ismail ${ }^{*}$, Alexander Schuh ${ }^{2}$ and Mohamed Abdul Hamed Ibrahim ${ }^{1}$ \\ ${ }^{1}$ Cairo University Hospital, Egypt \\ ${ }^{2}$ Musculoskeletal Centre Neumarkt,Neumarkt-Germany Musculoskeletal Center Neumarkt, Neumarkt Hospital, Germany
}

\begin{abstract}
Introduction: The aim of our study is to detect effectiveness of open carpal tunnel release (OCTR) versus endoscopic carpal tunnel release (ECTR) in diabetic patients, evaluate symptoms, functional improvement and wound healing post release.

Material and methods: During the period from 1/1/2014 till 1/4/2015 a randomized prospective double-blind trial was conducted in which 138 patients with diabetes and CTS was randomly divided into 2 groups A: for ECTR and B: for OCTR, mean follow up group A: 31.8 months and in group B: 31.7 months.

Results: There was significant improvement in functional scores in endoscopic versus open group and significant improvement in wound healing and less wound complications in ECTR group.
\end{abstract}

Conclusion: ECTR have a better outcome with better functional results than OCTR in diabetic patients with carpal tunnel release.

\section{Introduction}

Carpal tunnel syndrome (CTS) causes paresthesia, pain or numbness in the territory of the median nerve. Carpal tunnel syndrome is the most common entrapment neuropathy with a reported prevalence of $2 \%$ to $4 \%$ in the general population. Epidemiology studies have showed an increasing rate in CTS incidence, which is more prevalent in women and its ratio was estimated to be about 3-10:1. Diabetes mellitus is a known risk factor for developing carpal tunnel syndrome with a prevalence approaching $15 \%[1,2]$.

The physiological basis of this syndrome is related to ischemic events of the median nerve due to elevated internal canal pressure, which happens because of transverse carpal ligament (TCL) compression. Another hypothesis of this condition the development of tenosynovitis in the vicinity of this nerve. Although CTS usually occurs in the dominant hand, in some cases it happens in both. Some of the identified causes of CTS are collagen vascular diseases, metabolic disorders, pregnancy, and wrist dislocations or fractures, but in a large group of patients the reason remains idiopathic [1].

Diabetes is one of the leading causes of disability globally. Its incidence has been increasing because of population growth, population ageing and lifestyle changes. Previous studies have reported inconsistent results on the association between diabetes and CTS. Some studies found an increased risk of CTS in individuals with diabetes, a finding that was not confirmed by other studies. Moreover, it is unclear whether both Type 1 and Type 2 diabetes increase the risk of CTS [3].

The reason why CTS is more common in diabetes is not fully clarified. It has been suggested that a nerve, with already established endoneurial hypoxia caused by diabetes, could be more vulnerable to local compression. Additional mechanisms may involve increased concentrations of advanced glycation end products as well as myofibroblasts leading to synovitis and stiffening of the collagen tissue. Diabetes adversely affects health-related quality of life (HLQL). An even larger impact on HRQL is related to poor metabolic control, diabetic complications such as peripheral neuropathy, and the development of psychiatric symptoms [4].

Surgical treatment is performed when symptoms are severe or when conservative treatment fails. Traditional open carpal tunnel release (OCTR) is the gold standard for carpal tunnel decompression. This approach allows the surgeon to directly visualize the carpal tunnel and guarantees complete section. Endoscopic carpal tunnel release (ECTR) is a relatively new procedure that is much less invasive than OCTR. Two techniques are commonly used for ECTR. The first one is the single-portal technique designed by Agee; the other is the twoportal technique reported by Chow and Okutsu [5].

The aim of our study is to detect effectiveness of OCTR versus ECTR in diabetic patients, evaluate symptoms, functional improvement and wound healing post release.

\section{Material and method}

During the period from 1/1/2014 till 1/4/2015 a randomized prospective double blind therapeutic trial was conducted in which 138 patients (155 hands) with Diabetes mellitus and CTS was randomly

Correspondence to: Prof Dr. Mohamed Said Abdelmaksoud Mohamed Ismail Cairo University Hospital, Cairo, Egypt, E-mail: m.saidmaksoud@kasralainy.edu.eg

Key words: endoscopic, open, carpal tunnel syndrome, release, diabetes

Received: March 19, 2018; Accepted: April 02, 2018; Published: April 06, 2018 
divided into 2 groups using the opaque closed envelope technique group A: for endoscopic carpal tunnel release and group B: for open carpal tunnel release, all patients were given numbers, all patients was operated by same surgeon and follow up and result assessment done by the other 2 investigators in the trial to nullify the bias patients with bilateral affection were also given a number for each hand to be randomly allocated and operation was done 3 weeks after the first surgery 5 patients ( 10 hands) did OCTR 6 patients ( 12 hands) did ECTR and 6 patients ( 12 hands) did one side OCTR and the other side ECTR, there was no need for institutional review board approval as per institutional regulations.

\section{Inclusion criteria}

All patients with Diabetes mellitus and having CTS ranging from moderate to severe with no muscle atrophy, all patients were examined by same surgeon with positive Phalen's and Tinel's tests and positive NCS and all patients was having proper diabetic control.

\section{Exclusion criteria}

Patients with cervical radiculopathy, inflammatory arthropathy and chronic diseases (IHD, HTN) and uncontrolled diabetes were excluded from the study.

\section{Patient demographics}

The study included 138 patients with 155 hands 110 females and 28 males, 115 right hand and 40 left hands, mean age was 43.49 years (SD 6.93) in group A 39. 25 (SD5.11) in group B and the mean follow up period was 31.7 months (SD 4.5).

\section{Operation details and technique}

Operative technique in ECTR group was conducted as described by Steven m. Topper from Agee [6,7] and distal pillar incision in OCTR group (Figure 1).

\section{Anaesthesia}

In all patients the procedure was performed using local intravenous anaesthesia (Bier's block). None of them needed shifting to general anaesthesia.

\section{ECTR technique}

The surgical incision (Figure 2) is placed transversely in the proximal wrist crease between the flexor carpi ulnaris and the palmaris longus (PL), if the patient does not have a PL, the radial extent of the incision should be $2 \mathrm{~cm}$ radial to the flexor carpi ulnaris. The incision is usually $1 \mathrm{~cm}$ in length.

\section{Release}

The endoscope should hug the underside of the transverse carpal ligament (TCL) and use the leading edge of the device to push synovium out of the way. Transverse fibers of the ligament should be the only thing visualized in the viewing portal of the device (Figure 3). Defining the distal edge of the TCL is assisted by using a digit from the non-instrument hand to ballot in the area of the distal edge and it is usually $30 \mathrm{~mm}$ from the proximal edge of TCL defined at wound dissection. After release (Figure 4) the device is then reinserted to confirm complete division of the transverse carpal ligament.

\section{Closure}

Wound is closed with 2 simple stitches and $4 \mathrm{cc}$ of Marcaine is injected in subcutaneous area and the carpal tunnel. Elastic bandage is

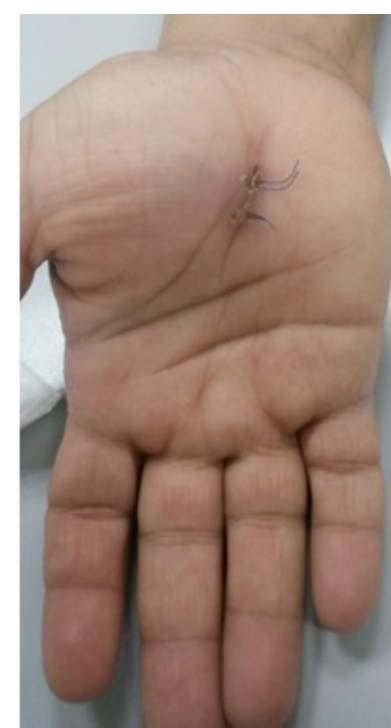

Figure 1. OCTR incision.

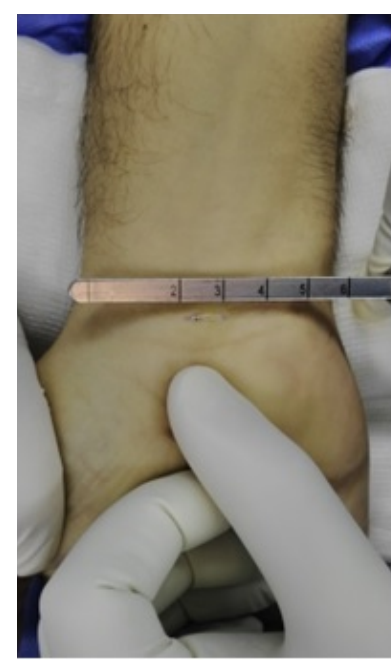

Figure 2. ECTR incision.

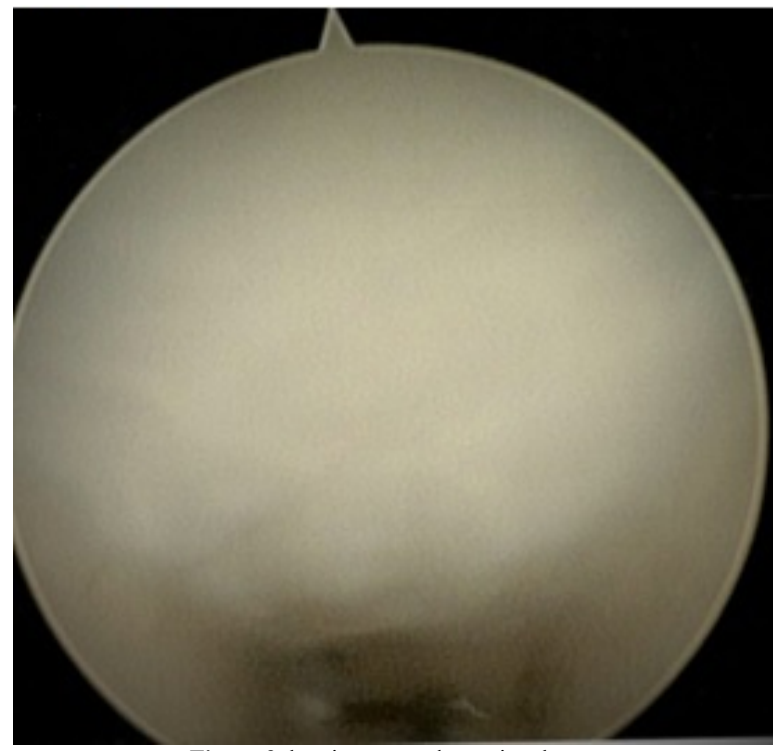

Figure 3. howing pre-endoscopic release. 


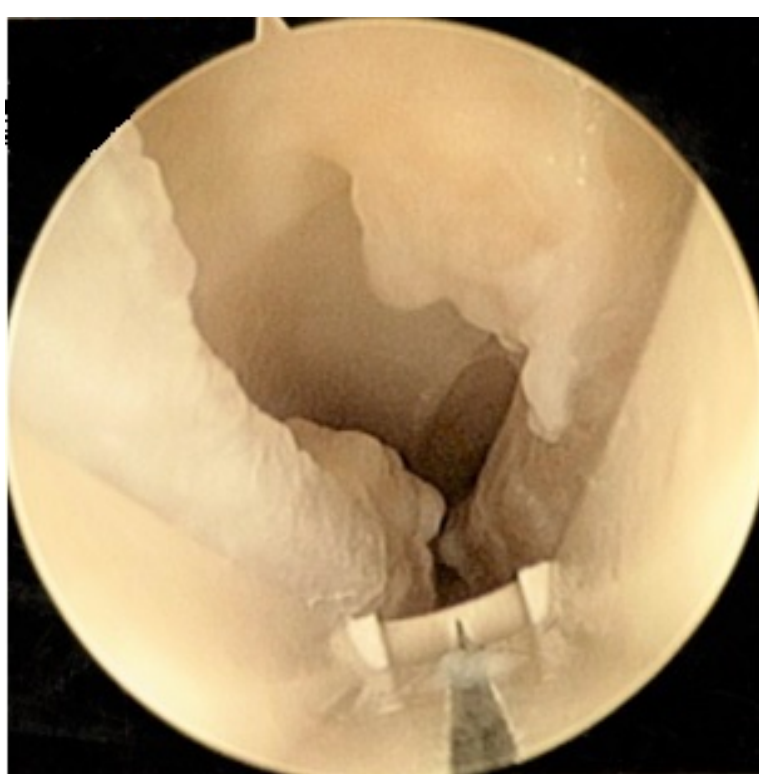

Figure 4. Showing post endoscopic release.

applied for 6 hours and removed and band aid is placed and all patients were discharged home the night of surgery following same principles from Duncan and flowers Discharge Criteria: Independent in all selfcare and dressing activities, 0-2 Weeks: Edema control PRN, Active/ passive ROM of digits, Scar massage, Patient able to use hand for bathing once sutures are removed [8].

\section{Operative time}

The mean operative time was 19.46 minutes in ECTR and 25.68 in OCTR group.

\section{Follow up}

Follow up was done in our outpatient hospital according to a standardized protocol in which all patients was instructed to visit the hospital after 2 week of operation for stitch removal and patient is allowed to return to office work once grip and pillar pain allows which was mean of 1.12 weeks in ECTR group and 2.7 weeks in OCTR group, Mean follow up period in group A: 31.8 months (SD 4.5) and in group B: 31.7 (SD 4.4), functional scores was assessed at 3,6,12 and 24 months, 140 patients are included with 15 patients lost in follow up period 8 in group a and 7 in group B, patients was assessed for any complications as: wound infection, persistent hand pain, weakness, RSD and pillar tenderness.

Analysis was done using SPSS version 22.0, Boston score was used to assess the improvement in symptoms and the functional outcome, in which 11 questions for symptoms improvement and 8 questions for functional outcome was assessed, all outcomes was assessed at 3,6,12 and 24 months post operatively.

Assessment of outcome was done by Boston score, period to return to work, pinch and grip function [9].

\section{Results}

The study included 155 patients ( 155 hands) 127 females and 28 males, 115 right hands and 40 left hands, mean age was 43.4 (SD 6.9) in group A and 39.3 (SD 5.1) group B, the mean follow up period was 31.7 months (SD 4.5), patients was randomly divided into 2 groups group A: for endoscopic carpal tunnel release and group B: for open carpal tunnel release, all patients were given numbers after allocation with 15 patients missed follow up post allocation.

Results assessment was according to Boston Score, return to work, pillar pain, pillar tenderness and period to regain full grip and pinch.

There was significant improvement in symptoms severity score (SSS) in group A with a mean of 1.46 (SD 0.14) than group B with a mean of 2.27 (SD 0.13) with p-value $<0.05$, there was significant improvement in hand pain day and night, hand dormancy day and night, tingling sensation and small object sensation in group A than group B.

Functional satisfactory score (FSS) showed significant improvement in group A with mean of 1.63 (SD 0.6) than group B with mean of 2.3 (SD 0.26) with $p$-value $<0.05$ and there was significant improvement in writing buttoning, holding small and large objects and self-care in group A than group B (Figure 5) shows difference in SSS and FSS in both groups, (Figure 6) show comparative results of SSS and FSS in both groups.

There was significant improvement in grip and pinch of objects in group A in a mean of 1.7 weeks (SD 0.25 ) than group B in a mean of 2.8 weeks (SD 0.6) with p value $<0.05$.

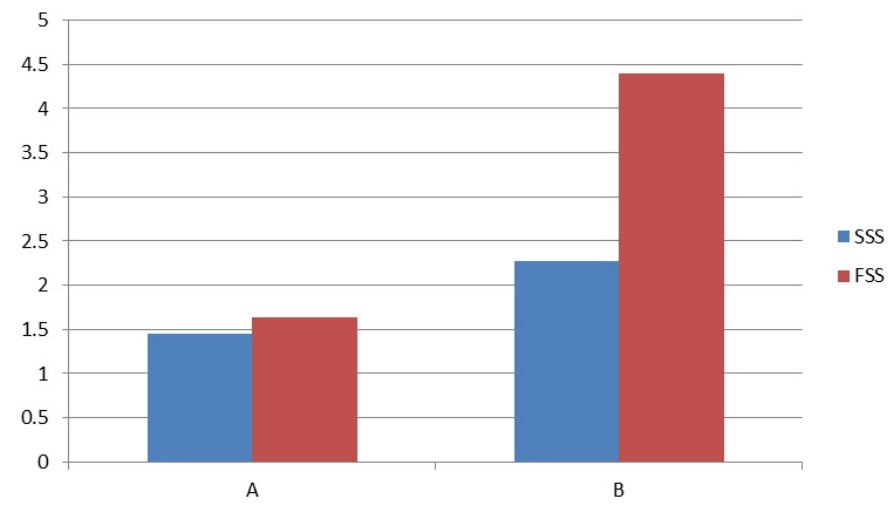

Figure 5. Difference in SSS and FSS in both groups.

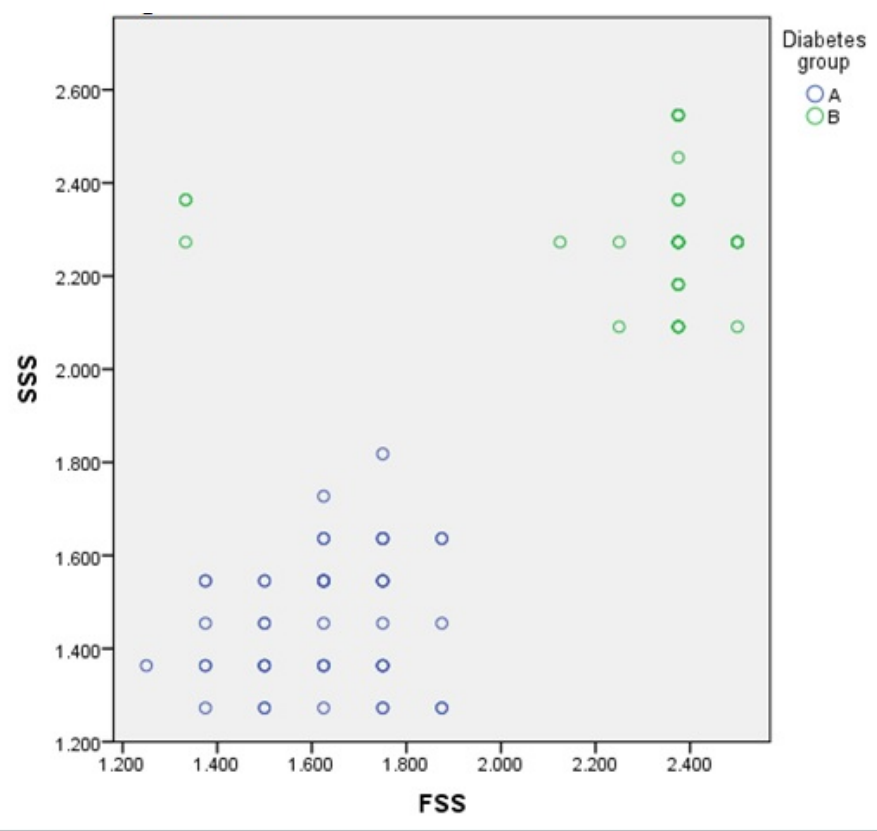

Figure 6. Shows SSS and FSS in both groups. 
Return to work showed significant improvement in group A with a mean of 1.2 weeks (SD 0.3) compared to a mean of 2.7 weeks (SD 0.6) in group $\mathrm{B}$ with $\mathrm{p}$ - value $<0.05$ (Figure 7 ).

There was significant improvement in wound healing and less wound complications in group A than group B, with mean wound healing period 8.17 days in group A and 18.2 days in group B, there were no cases of wound infection and dehiscence in group a with 18 cases of wound infection and delayed healing in group B. Figure 8 shows a patient with wound complication.

There was also significant correlation between age and improvement in FSS, SSS and return to work and there was significant correlation between improvement in FSS, SSS and return to work, pillar pain, pillar tenderness and grip and pinch improvement.

\section{Discussion}

Diabetes mellitus (DM) is a chronic systemic disorder with a wide range of complications. Moreover, DM is a common disease in all parts of the world and in recent years its incidence and significance has increased. Diabetic patients suffer from various rheumatic conditions that influence their quality of life [1].

The association between diabetes and carpal tunnel release was weaker than the association between diabetes and CTS. CTS is a complication of diabetes; therefore, carpal tunnel release may be

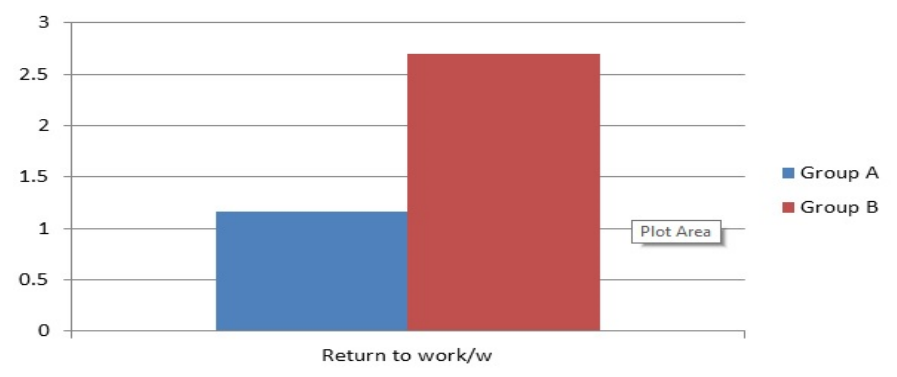

Figure 7. Return to work /W.

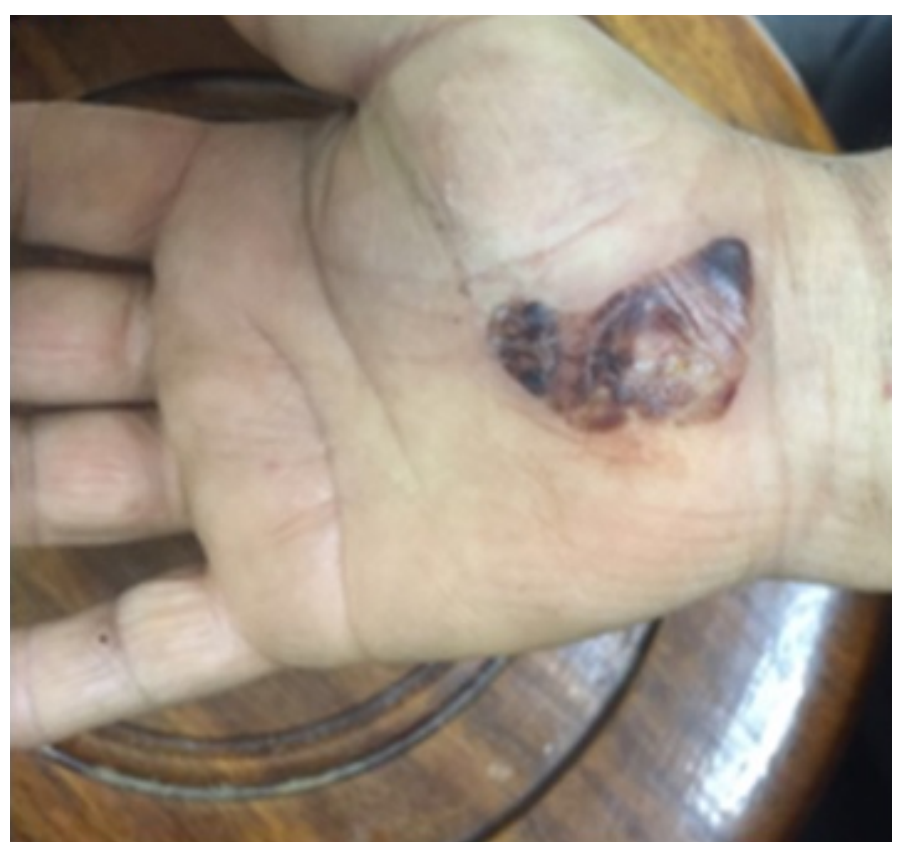

Figure 8. Post-operative wound complications in OCTR. recommended less often for patients with diabetes than for those without. The outcome of carpal tunnel release may be less favourable in patients with diabetes. In addition, in patients with diabetes, CTS coexists with Dupuytren's contracture, limited joint mobility or flexor tenosynovitis [3].

In a recent study Yucel $\mathrm{H}$. determined the associations between clinical, physical, and neurophysiological outcomes and self-reported symptoms and functions of the patients who had carpal tunnel release surgery. The BCTQ scores of our patients were associated with diabetes mellitus, migraine, lack of medical treatment, impaired light touch pressure sensation, pain and paresthesia at night, and fatigue symptoms. The presence of these factors in CTS adversely affected our patients' symptoms and functionality [10].

Gulabi et al. [11] indicated that diabetes mellitus is a risk factor of a poor functional outcome of carpal tunnel decompression.

Ozer K et al. [2] indicated a high level of satisfaction among diabetic patients following carpal tunnel release. Both functional and sensory Boston carpal tunnel questionnaire (BCTQ) scales showed significant change in scores after surgery, the results indicate that the surgery for diabetics is satisfactory in $68 \%$ of the patients, the strengths of this study include prospective design, operations performed by a single surgeon and the use of patient-reported outcome questionnaire avoiding bias. Its limitations include the smaller sample size for diabetic patients, and we did not factor in the presence of diabetic neuropathy [2].

Thomson et al. report firstly on health-related quality of life (HRQL) in patients with diabetes, based on a follow-up of a minimum of 5-years, after carpal tunnel release. The domains bodily pain and role physical have previously been proved reliable, and sensitive in detecting changes after carpal tunnel release. In accordance, the study was able to demonstrate a marked improvement in both role physical and bodily pain after surgery. As this improvement was maintained for both patient's groups at the 5-year follow-up, we consider that the marked reduction in physical functioning in the patients with diabetes is caused by disorders other than CTS [12].

Cederlund et al. concluded that results after carpal tunnel release have been rewarding, with reported success rates above $80 \%$. In contrast, it has often been stated that patients with diabetes and CTS achieve less predictable results with uncertain sensory and motor recovery. In support of such a statement, nerve conduction velocity and vibrotactile sense, reflecting large nerve fibre function, has been demonstrated to be significantly impaired in patients with diabetes before and after carpal tunnel release compared with patients without diabetes [13].

The findings of a meta-analysis by Sayegh ET and Strauch RJ [14] suggest that symptom relief and clinical outcomes, according to the validated BCTQ symptom severity and functional status indices, were no different for patients treated with endoscopic versus open release, Endoscopic release provides improved grip and pinch strength during the early postoperative period, an earlier return to work, and a lower risk of scar tenderness. Return-to-work data are difficult to interpret because patients often return to work when indicated by their surgeon, regardless of procedure type. However, superior improvement of the grip and pinch strength with endoscopic release during the early postoperative period compared with open release may explain why patients undergoing endoscopic release can return to work sooner [14].

Means et al. [15] postulated after a follow-up period of 8 years that patients had significantly improved average symptom severity and functional scores at long-term intervals compared with their baseline 
preoperative scores. Our results highlight the potentially low incidence of carpal tunnel syndrome recurrence in the long-term follow-up period for this surgical technique [16]. Another study by Louie et al. [17] showed that ECTR generally provides strong outcomes while carrying a low, but undeniable, risk of recurrence, and indicates that long-term results are essentially indistinguishable between ECTR and OCTR, so the choice of procedure is a matter of surgeon preference. Kohanzadeh et al. [15] stated that the learning curve for each of the methods must be taken into consideration when evaluating results and outcome, both techniques require proper training and experience before proficiency is reached [15].

Our study is a prospective double blind trial that investigates the results of outcome between ECTR and OCTR on diabetic patients, the results of our study revealed significantly better outcome in patients treated with ECTR compared with the results of patients treated by OCTR, ECTR group having better relief in symptoms and better function scores, less pillar pain and tenderness at 12 weeks postoperative, faster regain of grip and pinching functions, significantly faster return to work and significant improvement in wound healing and significant reduction in wound infection and complications.

The strength of our study is that it is prospective randomized double blind controlled trial and limitations were the sample size and patients missed in follow up.

\section{Conclusion}

ECTR have a better outcome with better functional results than OCTR in diabetic patients with carpal tunnel release.

\section{Approved from esthical commettie cairo university}

\section{Acknowledgment}

Dr. Ahmad Samir Alfaar: MB.BCh (MD),MSc (Oph), MSc (Onc), Dip Med. Inf. Charité - Universitätsmedizin Berlin, Statistical analysis of the results.

Conflict of Interest: The authors declare that they have no conflict of interest.

Funding: There is no funding source.

Ethical approval: Approved by the ethical committee of University hospital

\section{References}

1. Ebrahimzadeh MH, Mashhadinejad H, Moradi A, Kachooei AR (2013) Carpal Tunnel Release in Diabetic and Non-Diabetic Patients. Arch Bone Jt Surg 1: 23-27. [Crossref]

2. Ozer K, Malay S, Toker S, Chung KC (2013) Minimal clinically important difference of carpal tunnel release in diabetic and nondiabetic patients. Plast Reconstr Surg. 131: 1279-1285. [Crossref]

3. Pourmemari MH, Shiri R (2016) Diabetes as a risk factor for carpal tunnel syndrome: systematic review and meta-analysis. Diabet Med 33: 10-16. [Crossref]

4. Thomsen NO, Björk J, Cederlund RI (2014) Health-related quality of life 5 years after carpal tunnel release among patients with diabetes: a prospective study with matched controls. BMC Endocr Disord 14:85. [Crossref]

5. Chen L, Duan X, Huang X, Lv J, Peng K, et al. (2014) Effectiveness and safety of endoscopic versus open carpal tunnel decompression. Arch Orthop Trauma Surg 134: 585-593. [Crossref]

6. Agee JM, McCarroll HR, North ER (1994) Endoscopic carpal tunnel release using the single proximal incision technique. Hand Clin 10: 647-659. [Crossref]

7. Topper SM (2015) Endoscopic Carpal Tunnel Release. In W. B. Geissler (Ed.),Wrist and Elbow Arthroscopy. Springer New York: 341-347.

8. Duncan SFM, Flowers C (2015) Carpal Tunnel Release. In: Therapy of the Hand and Upper Extremity. Rehabilitation Protocols. Springer New York: 153-156.

9. Meirelles L M, Santos JB, Santos LL (2006) Evaluation of Boston questionnaire applied at late post-operative period of carpal tunnel syndrome operated with the paine retinaculatome through palmar port. Acta ortop Bras 14: 126-132.

10. Yucel H (2015) Factors affecting symptoms and functionality of patients with carpal tunnel syndrome: a retrospective study. J Phys Ther Sci 27: 1097-1101. [Crossref]

11. Gulabi D, Cecen G, Guclu B, Cecen A (2014) Carpal tunnel release in patients with diabetes result in poorer outcome in long-term study. Eur J Orthop Surg Traumatol 24: 1181-1184.

12. Thomsen NO, Cederlund RI, Andersson GS, Rosén I, Björk J, et al. (2014) Carpal tunnel release in patients with diabetes: a 5-year follow-up with matched controls. $J$ Hand Surg Am 39: 713-720. [Crossref]

13. Cederlund RI, Dahlin LB, Thomsen NO (2012) Activity limitations before and after surgical carpal tunnel release among patients with and without diabetes. $J$ Rehabil Med 44: 261-267. [Crossref]

14. Sayegh ET, Strauch R J (2015) Open versus Endoscopic Carpal Tunnel Release: A Meta-analysis of Randomized Controlled Trials. Clin Orthop Relat Res 473: 11201132. [Crossref]

15. Kohanzadeh S, Herrera FA, Dobke M (2012) Outcomes of open and endoscopic carpal tunnel release: a meta-analysis. Hand 7: 247-251. [Crossref]

16. Means KR, Dubin NH, Patel KM, Pletka JD (2014) Long-term outcomes following single-portal endoscopic carpal tunnel release. Hand 9: 384-388. [Crossref]

17. Louie D, Earp B, Blazar P (2012) Long-term outcomes of carpal tunnel release: a critical review of the literature. Hand 7: 242-246. [Crossref]

Copyright: (C2018 Ismail MSAM. This is an open-access article distributed under the terms of the Creative Commons Attribution License, which permits unrestricted use, distribution, and reproduction in any medium, provided the original author and source are credited. 